NUMBER THEORY WEEK 2017

BANACH CENTER PUBLICATIONS, VOLUME 118

INSTITUTE OF MATHEMATICS

POLISH ACADEMY OF SCIENCES

WARSZAWA 2019

\title{
ON LARGE VALUES OF HARDY'S FUNCTION $Z(t)$ AND ITS DERIVATIVES
}

\author{
ALEKSANDAR IVIĆ \\ Serbian Academy of Arts and Sciences \\ Knez Mihailova 35, 11000 Beograd, Serbia \\ E-mail: aleksandar.ivic@rgf.bg.ac.rs,aivic_2000@yahoo.com
}

Abstract. Let $Z(t)=\zeta\left(\frac{1}{2}+i t\right) \chi^{-1 / 2}\left(\frac{1}{2}+i t\right)$ denote as usual Hardy's function, where $\zeta(s)=$ $\chi(s) \zeta(1-s)$ is the functional equation for the Riemann zeta-function $\zeta(s)$. It is proved that, for $t \geqslant t_{0}>0$,

$$
\begin{array}{cc}
\max _{T \leqslant t \leqslant T+H, Z(t)>0} Z(t) \gg(\log T)^{1 / 4} & \left(T^{\theta+\varepsilon} \leqslant H \leqslant T\right), \\
\max _{T \leqslant t \leqslant T+H, Z(t)<0}-Z(t) \gg(\log T)^{1 / 4} & \left(T^{\theta+\varepsilon} \leqslant H \leqslant T\right),
\end{array}
$$

where $\theta=\frac{17}{110}=0.15 \overline{45}$. A similar result is shown for large values of $Z^{(k)}(t)$, where $k \geqslant 1$ is a fixed integer. Several related topics are also discussed.

1. Introduction and statement of results. Let the Riemann zeta-function be, as usual,

$$
\zeta(s)=\sum_{n=1}^{\infty} n^{-s} \quad(\Re s>1) .
$$

For $\Re s \leqslant 1$ one defines $\zeta(s)$ by analytic continuation (see the monographs of H. M. Edwards [8], the author [14], [16] and E. C. Titchmarsh [27] for the properties of $\zeta(s))$. Bounds for large values of $|\zeta(s)|$, and in particular for $\left|\zeta\left(\frac{1}{2}+i t\right)\right|$, have been always of great interest. A. Bondarenko and K. Seip [6] recently proved the following result. Let $0<\beta<1$ be given and let $c$ satisfy

$$
0<c<\sqrt{\min (1 / 2,1-\beta)} .
$$

2010 Mathematics Subject Classification: Primary 11M06.

Key words and phrases: Riemann zeta-function, Hardy's function $Z(t)$ and its derivatives, large values, exponent pairs.

The paper is in final form and no version of it will be published elsewhere. 
Then, for $T \geqslant T_{0}(>0)$, there exists $t \in\left[T^{\beta}, T\right]$ such that

$$
\left|\zeta\left(\frac{1}{2}+i t\right)\right| \geqslant \exp \left(c \sqrt{\frac{\log T \log \log \log T}{\log \log T}}\right) .
$$

The lower bound in (1.1) improves, by a factor of $\sqrt{\log \log \log T}$ in the exponential, on previous results of H. L. Montgomery [24, R. Balasubramanian [2], R. BalasubramanianK. Ramachandra [25] and K. Soundararajan [26]. In particular, R. BalasubramanianK. Ramachandra ([25], Th. 3.2.1) stated the more general result that

$$
\max _{T \leqslant t \leqslant T+H}\left|\zeta\left(\frac{1}{2}+i t\right)\right| \geqslant \exp \left(B \sqrt{\frac{\log H}{\log \log H}}\right) \quad(\log \log T \ll H \leqslant T)
$$

with $B=0.75$. In [26] K. Soundararajan indicated that the method actually allows $B=0.530 \ldots$ in 1.2 .

On the other hand, it seems also interesting to investigate the occurrence of large values of Hardy's function (see the author's monograph [19] for its properties)

$$
Z(t):=\zeta\left(\frac{1}{2}+i t\right)\left(\chi\left(\frac{1}{2}+i t\right)\right)^{-1 / 2}, \quad \zeta(s)=\chi(s) \zeta(1-s),
$$

so that

$$
\chi(s)=\frac{\Gamma\left(\frac{1}{2}(1-s)\right)}{\Gamma\left(\frac{1}{2} s\right)} \pi^{s-1 / 2} .
$$

It follows that $Z(t)$ is a smooth, real-valued function of the real variable $t$, for which

$$
|Z(t)|=\left|\zeta\left(\frac{1}{2}+i t\right)\right|
$$

Thus the bound in 1.1 ( or 1.2$)$ ) is attained either for $Z(t)$ or for $-Z(t)$, but one cannot say for which. Hence it seems of interest to find large positive values of $Z(t)$ in $[T, T+H]$ for suitable $0<H \leqslant T$, or small negative values in the same interval. No results seem to exist in this direction, and the methods used in obtaining (1.1) and (1.2) do not work here. Some results about the distribution of positive and negative values of $Z(t)$ were recently obtained by S. M. Gonek and the author [9]. In particular, it was proved there that

$$
\mu\left(I_{+}^{(0)}(T, T)\right) \gg T \quad \text { and } \quad \mu\left(I_{-}^{(0)}(T, T)\right) \gg T
$$

where $\mu(\cdot)$ denotes the Lebesgue measure and, for a fixed integer $k \geqslant 0$,

$$
\begin{aligned}
& I_{+}^{(k)}(T, H)=\left\{t:(T \leqslant t \leqslant T+H) \wedge\left(Z^{(k)}(t)>0\right)\right\}, \\
& I_{-}^{(k)}(T, H)=\left\{t:(T \leqslant t \leqslant T+H) \wedge\left(Z^{(k)}(t)<0\right)\right\} .
\end{aligned}
$$

In this work we shall obtain some results on large values of $Z^{(k)}(t)$ (the $k$-th derivative of $Z(t)$ ) and some related topics. The symbol $\ll_{\varepsilon}$ will mean that the «-constant depends on $\varepsilon$, an arbitrarily small, positive constant, not necessarily the same one at each occurrence. 
THEOREM 1. For $T \geqslant T_{0}>0$ we have

$$
\begin{array}{r}
\max _{t \in I_{+}^{(0)}(T, H)} Z(t)=\max _{T \leqslant t \leqslant T+H, Z(t)>0} Z(t) \gg(\log T)^{1 / 4} \quad\left(T^{\theta+\varepsilon} \leqslant H \leqslant T\right), \\
\max _{t \in I_{-}^{(0)}(T, H)}(-Z(t))=\max _{T \leqslant t \leqslant T+H, Z(t)<0}(-Z(t)) \gg(\log T)^{1 / 4} \quad\left(T^{\theta+\varepsilon} \leqslant H \leqslant T\right),
\end{array}
$$

where

$$
\theta=\frac{17}{110}=0.15 \overline{45}
$$

TheOREM 2. For $k \geqslant 1$ a fixed integer let $\theta_{1}=\min \left(\frac{3}{37}, \mu\left(\frac{1}{2}\right)\right)+\varepsilon$, and

$$
\theta_{k}=\min \left\{\frac{2 \kappa+2 \lambda-1}{4 k+8 \kappa+4 \lambda}+\varepsilon, \frac{1}{k} \mu\left(\frac{1}{2}\right)+\varepsilon\right\} \quad(k \geqslant 2) .
$$

Here $\mu(\sigma)=\limsup _{t \rightarrow \infty} \log |\zeta(\sigma+i t)| / \log t$, and $(\kappa, \lambda)$ is an exponent pair. Then, for $T^{\theta_{k}} \leqslant H \leqslant T$, we have

$$
\begin{array}{r}
\max _{t \in I_{+}^{(k)}(T, H)} Z^{(k)}(t) \gg \log ^{k} T, \\
\max _{t \in I_{-}^{(k)}(T, H)}\left(-Z^{(k)}(t)\right) \gg \log ^{k} T .
\end{array}
$$

Note that Theorem 2 is a sort of generalization of Theorem 1.

THEOREM 3. Let $\zeta\left(\frac{1}{2}+i T\right)=0$, and let $Z(t)$ be monotonic in $[T, T+H]$ for some $H=H(T)$ satisfying $T^{\varepsilon} \leqslant H \leqslant T^{1 / 6}$. Then, for $(\kappa, \lambda)$ as before and $T \leqslant t \leqslant T+3 H / 4$,

$$
\zeta\left(\frac{1}{2}+i t\right) \ll_{\varepsilon} T^{(\kappa+\lambda) / 2-1 / 4+\varepsilon} H^{-2 \kappa-\lambda} .
$$

This result essentially says that, if the difference between two consecutive zeros of $Z(t)$ is large, then $\left|\zeta\left(\frac{1}{2}+i t\right)\right|$ between these zeros is relatively small. To assess the strength of [1.8, we can take, e.g., the exponent pair (2.6) in (1.8). Then we obtain

$$
\zeta\left(\frac{1}{2}+i t\right) \ll_{\varepsilon} T^{13 / 84+\varepsilon} H^{-81 / 84} \quad\left(T \leqslant t \leqslant T+3 H / 4, \zeta\left(\frac{1}{2}+i T\right)=0\right)
$$

and $Z(t)$ is monotonic in $[T, T+H]$. In particular, if these conditions are satisfied, then with $H=T^{1 / 81}$ it follows that

$$
\zeta\left(\frac{1}{2}+i t\right) \ll_{\varepsilon} T^{1 / 7+\varepsilon} \quad(1 / 7=0 . \overline{142857}) .
$$

The bound 1.10 is still not known to hold unconditionally (see (2.7)). Even this is far from the (yet unproved) Lindelöf Hypothesis that $\zeta\left(\frac{1}{2}+i t\right) \ll_{\varepsilon} t^{\varepsilon}$.

2. Exponent pairs and a special exponential sum. The proofs of all three theorems reduce to the estimation of a special type of exponential sum, namely

$$
S=S(M, T):=\sum_{M<m \leqslant M^{\prime} \leqslant 2 M} \exp (i T \log (Q-m)), \quad Q=\left[(T /(2 \pi))^{1 / 2}\right],
$$

where $[x]$ is the greatest integer not exceeding $x, M^{\prime}$ is a fixed integer in the range $M<M^{\prime} \leqslant 2 M, M$ assumes one of the $O(\log T)$ values $M=T^{1 / 2+\varepsilon} H^{-1} 2^{-j}, j=1,2, \ldots$, and $T^{\varepsilon} \leqslant H \leqslant T^{1 / 6}$. Here one can take advantage of the fact that the exponential sum 
in 2.1 is of a special nature, since $n=Q-m$ is close to $(T /(2 \pi))^{1 / 2}$. This idea was already exploited by A. A. Karatsuba [21], and in the author's works [14, [15] and [16]. For the sake of completeness, it will be given here in some detail.

If $Q=\left[(T /(2 \pi))^{1 / 2}\right]$, then for some $\theta$ satisfying $0 \leqslant \theta<1$ we have $T=2 \pi(Q+\theta)^{2}$. Therefore

$$
\begin{aligned}
T \log (Q-m)-T \log Q= & -T \sum_{j=1}^{\infty}(m / Q)^{j} j^{-1} \\
= & -2 \pi Q m-2 \pi\left(2 Q \theta+\theta^{2}\right) m Q^{-1}-\pi m^{2} \\
& -2 \pi\left(2 Q \theta+\theta^{2}\right) m^{2}\left(2 Q^{2}\right)^{-1}-T\left(\frac{m^{3}}{3 Q^{3}}+\frac{m^{4}}{4 Q^{4}}+\ldots\right) .
\end{aligned}
$$

Taking into account that $\exp (2 \pi i r)=1$ for any integer $r$ and considering separately even and odd $m$ (to get rid of the term $\pi m^{2}$ in the above expression) we obtain

$$
|S| \leqslant\left|S^{\prime}\right|+\left|S^{\prime \prime}\right|
$$

where both sums $S^{\prime}$ and $S^{\prime \prime}$ are estimated analogously. Here $S^{\prime}$ arises from even values of $m$ and equals

$$
S^{\prime}=\sum_{M_{1}<m \leqslant M_{1}^{\prime} \leqslant 2 M_{1}} \exp (2 \pi i f(m)) \quad\left(M \ll M_{1} \ll M\right),
$$

where we set

$$
\begin{gathered}
f(x)=b_{1} x+b_{2} x^{2}+\frac{T}{2 \pi}\left(\frac{(2 x)^{3}}{3 Q^{3}}+\frac{(2 x)^{4}}{4 Q^{4}}+\ldots\right), \\
b_{1}=2\left(2 Q \theta+\theta^{2}\right) Q^{-1} \ll 1, \quad b_{2}=b_{1} Q^{-1} \ll Q^{-1} .
\end{gathered}
$$

If $1 \ll M \ll T^{1 / 4}$, then we use Lemma 2.6 (with $k=3, K=2^{k-1}=4, \lambda_{3}=T^{-1 / 2}$ ) of [14] to obtain $S \ll T^{5 / 24}$. If $M \gg T^{1 / 4}$ and $M_{1} \leqslant x \leqslant 2 M_{1}$, then we have $f^{\prime}(x) \gg 1$ and, as $x \rightarrow \infty$,

$$
\begin{aligned}
& f^{(k)}(x)=\left(c_{k}+o(1)\right) x^{3-k} T^{-1 / 2} \quad(k \leqslant 3), \\
& f^{(k)}(x)=\left(c_{k}+o(1)\right) T^{1-k / 2} \quad(k>3) .
\end{aligned}
$$

In this case we shall estimate $S^{\prime}$ by van der Corput's classical theory of exponent pairs. This method explained in detail in Chapter 2 of [14. The precise (technical) definition of an exponent pair is given there on top of p. 80, but in many instances (including (2.2p) it suffices to have $f(x) \in C^{r}[B, 2 B]$ for some $r \geqslant 5$, and moreover the derivatives of $f(x)$ for $x \in[B, 2 B]$ and $r=1,2, \ldots$ satisfy

$$
A B^{1-r} \ll_{r}\left|f^{(r)}(x)\right| \ll_{r} A B^{1-r} .
$$

If $A \ll\left|f^{\prime}(x)\right| \ll A, A>1 / 2$ then $(\kappa, \lambda)$ is an exponent pair if $0 \leqslant \kappa \leqslant \frac{1}{2} \leqslant \lambda \leqslant 1$ and

$$
\sum_{B<n \leqslant B+h} \mathrm{e}(f(n)) \ll A^{\kappa} B^{\lambda} \quad(B \geqslant 1,1<h \leqslant B, \mathrm{e}(x)=\exp (2 \pi i x)) .
$$

Trivially $(0,1)$ is an exponent pair, and if $(\kappa, \lambda)$ is an exponent pair, so is also

$$
(k, \ell)=\left(\frac{\kappa}{2 \kappa+2}, \frac{1}{2}+\frac{\lambda}{2 \kappa+2}\right), \quad(k, \ell)=\left(\lambda-\frac{1}{2}, \kappa+\frac{1}{2}\right) .
$$


The first process of forming new exponent pairs is called the $A$-process, and the second one the $B$-process, as shown in Lemma 2.8 and Lemma 2.9 of [14]. Additionally the set of exponent pairs is trivially a convex set of two-dimensional points, and the process of forming exponent pairs through convexity is called the $C$-process.

There are, however, possibilities to construct exponent pairs which do not arise from the trivial one by applying the $A$-, $B$ - or $C$-process. Historically the first such pair was

$$
\left(\kappa_{1}, \lambda_{1}\right)=\left(\frac{9}{56}+\varepsilon, \frac{37}{56}+\varepsilon\right),
$$

obtained in the pioneering works of E. Bombieri and H. Iwaniec [4], [5]. The more recent work on this subject was done by M. N. Huxley (see e.g., [11, [12, [13]), who elaborated on the ideas of Bombieri-Iwaniec. The new exponent pairs were obtained by refining the original Bombieri-Iwaniec method. J. Bourgain [7] recently showed that

$$
\left(\kappa_{0}, \lambda_{0}\right)=\left(\frac{13}{84}+\varepsilon, \frac{55}{84}+\varepsilon\right)
$$

is another exponent pair which does not arise by the use of classical theory. Both exponent pairs in 2.5) and 2.6 have $\lambda=\kappa+1 / 2$, but $\kappa_{0}<\kappa_{1}$. In [7] Bourgain also proved that

$$
\zeta\left(\frac{1}{2}+i t\right) \ll_{\varepsilon}|t|^{13 / 84+\varepsilon}, \quad \frac{13}{84}=0.154761 \ldots,
$$

which is currently the best result of its kind. Note that 53/342 $>\theta=17 / 110$ of Theorem 1 .

The function $f(x)$ in 2.2 does not satisfy directly the definition of exponent pairs, although $f^{(k)}(x) \ll_{k} x^{3-k} T^{-1 / 2}$ for $k>3$, which is also satisfied for $1 \leqslant k \leqslant 3$. But, as explained in [15], if we apply the $A$-process and then the $B$-process to the exponential sum $S^{\prime}$, we obtain an exponential sum to which the theory of exponent pairs may be applied. In other words, if $(\kappa, \lambda)=B A(p, q)$ for an exponent pair $(p, q)$, then since the order of $f^{\prime}(x)$ is $M^{2} T^{-1 / 2}$, it follows that

$$
S^{\prime} \ll\left(M^{2} T^{-1 / 2}\right)^{\kappa} M^{\lambda} \quad\left(T^{1 / 4} \ll M \ll T^{1 / 2}\right) .
$$

Thus, in the whole range for $M$,

$$
\left|S^{\prime}\right|+\left|S^{\prime \prime}\right| \ll M^{2 \kappa+\lambda} T^{-\kappa / 2}+T^{5 / 24} .
$$

Therefore the sum in 2.1 satisfies

$$
S \ll_{\varepsilon} T^{\varepsilon}\left(\left(T^{1 / 2} H^{-1}\right)^{2 \kappa+\lambda} T^{-\kappa / 2}+T^{5 / 24}\right) .
$$

3. Proof of Theorem 1. Suppose that $0<H \leqslant T$ and that $k \geqslant 0$ is a fixed integer. The first step in both the proofs of Theorem 1 and Theorem 2 is to observe that, in view of 1.3 ,

$$
\begin{aligned}
\int_{T}^{T+H} Z^{(k)}(t) \varphi(t) \mathrm{d} t & =\int_{I_{+}^{(k)}(T, H)} Z^{(k)}(t) \varphi(t) \mathrm{d} t+\int_{I_{-}^{(k)}(T, H)} Z^{(k)}(t) \varphi(t) \mathrm{d} t, \\
\int_{T}^{T+H}\left|Z^{(k)}(t)\right| \varphi(t) \mathrm{d} t & =\int_{I_{+}^{(k)}(T, H)} Z^{(k)}(t) \varphi(t) \mathrm{d} t-\int_{I_{-}^{(k)}(T, H)} Z^{(k)}(t) \varphi(t) \mathrm{d} t .
\end{aligned}
$$


Here $\varphi(t)$ is a smooth, nonnegative function, supported in $[T, T+H]$, such that $\varphi(t)=1$ when $T+H / 4 \leqslant t \leqslant T+3 H / 4$. For an explicit construction of such a function, see e.g., Lemma 4.3 of the author's monograph [16. We have

$$
\varphi^{(r)}(t) \ll_{r} H^{-r} \quad(r=0,1,2, \ldots) .
$$

On adding the two expressions in 3.1 it follows that

$$
\int_{I_{+}^{(k)}(T, H)} Z^{(k)}(t) \varphi(t) \mathrm{d} t=\frac{1}{2}\left(\int_{T}^{T+H} Z^{(k)}(t) \varphi(t) \mathrm{d} t+\int_{T}^{T+H}\left|Z^{(k)}(t)\right| \varphi(t) \mathrm{d} t\right),
$$

and subtracting them one has

$$
\int_{I_{-}^{(k)}(T, H)} Z^{(k)}(t) \varphi(t) \mathrm{d} t=\frac{1}{2}\left(\int_{T}^{T+H} Z^{(k)}(t) \varphi(t) \mathrm{d} t-\int_{T}^{T+H}\left|Z^{(k)}(t)\right| \varphi(t) \mathrm{d} t\right) .
$$

The proofs of both bounds in (1.4) are analogous, so only the first one will be considered in detail. We remark that the sets in $(1.3)$ are non-empty in view of (3.4) and (3.5) below. We start from 3.3 with $k=0$, noting that, for $\log \log T \ll H \leqslant T$,

$$
\int_{T}^{T+H}|Z(t)| \varphi(t) \mathrm{d} t \geqslant \int_{T+H / 4}^{T+3 H / 4}\left|\zeta\left(\frac{1}{2}+i t\right)\right| \mathrm{d} t \gg H(\log H)^{1 / 4} .
$$

For the second lower bound inequality in (3.4) see e.g., K. Ramachandra [25]. If we can prove that, for $H=T^{\theta+\varepsilon}$ with $\theta$ given by 1.5 ,

$$
\int_{T}^{T+H} Z(t) \varphi(t) \mathrm{d} t \ll H,
$$

then from 3.3 with $k=0,3.4$ and 3.5 it follows that

$$
H \max _{T \leqslant t \leqslant T+H, Z(t)>0} Z(t) \geqslant \int_{T, Z(t)>0}^{T+H}|Z(t)| \varphi(t) \mathrm{d} t \gg \int_{T}^{T+H}|Z(t)| \varphi(t) \mathrm{d} t \gg H(\log H)^{1 / 4} .
$$

This gives the first bound in 1.4 , if $T^{\theta+\varepsilon} \leqslant H \leqslant T$. Namely if this bound holds with $H=T^{\theta+\varepsilon}$, it obviously holds for larger $H$ as well.

To see how one obtains 3.5 , with $H$ as in Theorem 1, one proceeds as in Section 10.3 of [14] or Section 2.4 of [16], using a weak form of the so-called Riemann-Siegel formula for $Z(t)$, namely the asymptotic formula

$$
Z(t)=2 \sum_{n \leqslant \sqrt{t /(2 \pi)}} n^{-1 / 2} \cos \left(t \log \frac{\sqrt{t /(2 \pi)}}{n}-\frac{t}{2}-\frac{\pi}{8}\right)+O\left(t^{-1 / 4}\right) .
$$

We restrict first $H$ to the range $0<H \leqslant T^{1 / 4}$, and insert 3.6 in the integral in 3.5 . Consider the terms for which $|n-\sqrt{t /(2 \pi)}| \geqslant 2 T^{1 / 2+\varepsilon} H^{-1}$. It follows that one has then $|n-\sqrt{T /(2 \pi)}| \geqslant T^{1 / 2+\varepsilon} H^{-1}$, in view of our range for $H$. Then each integration by parts of these terms in (3.5) leads to a similar integral as before, which is smaller by a factor which is $\ll T^{-\varepsilon}$. After integrating sufficiently many times by parts, with the aid of 3.2 , 
it is seen that these make a contribution which is $\ll_{\varepsilon} 1$, namely negligible. Analogously to $(10.37)$ of 14 one obtains

$$
\begin{aligned}
\int_{T}^{T+H} Z(t) \varphi(t) \mathrm{d} t & \ll_{\varepsilon} H T^{\varepsilon-1 / 4} \\
& +H T^{\varepsilon-1 / 4} \sum_{M} \max _{M^{\prime}}\left|\sum_{M<m \leqslant M^{\prime}<2 M} \exp (i T \log (Q-m))\right|
\end{aligned}
$$

where $Q=\left[(T /(2 \pi))^{1 / 2}\right]$, and $\sum_{M}$ denotes summation over $O(\log T)$ values

$$
M=2^{-j} T^{1 / 2+\varepsilon} H^{-1}, \quad j=1,2, \ldots .
$$

The exponential sum in $(3.7)$ is the same as the one in 2.1). Thus we may use 2.8 to deduce that the integral in (3.7) is

$$
\ll_{\varepsilon} H T^{\varepsilon-1 / 4}\left(T^{5 / 24}+\left(T^{1 / 2} H^{-1}\right)^{2 \kappa+\lambda} T^{-\kappa / 2}\right) \ll_{\varepsilon} H
$$

if

$$
H=T^{(2 \kappa+2 \lambda-1) /(8 \kappa+4 \lambda)+\varepsilon} .
$$

Choosing $(\kappa, \lambda)=A\left(\kappa_{0}, \lambda_{0}\right)$, with $\left(\kappa_{0}, \lambda_{0}\right)$ given by 2.6$)$, we have

$$
\frac{2 \kappa+2 \lambda-1}{8 \kappa+4 \lambda}=\frac{\kappa_{0}+\lambda_{0}}{4\left(\kappa_{0}+\lambda_{0}\right)+2} .
$$

Therefore with the exponent pair 2.6) we obtain $\theta=17 / 110+\varepsilon$, as asserted by 1.5 .

Note that we can take $\theta_{1} \equiv \theta=17 / 110+\varepsilon$ in $(2.1)$. The previous value $\theta_{1}$ arose from the exponent pair

$$
(\kappa, \lambda)=\left(\frac{1}{2} \alpha+\varepsilon, \frac{1}{2}+\frac{1}{2} \alpha+\varepsilon\right), \quad \alpha=0.329021356 \ldots,
$$

which minimizes $\kappa+\lambda$ by the use of classical processes for the creation of exponent pairs. One has then

$$
\frac{\kappa+\lambda}{4(\kappa+\lambda)+2}=\theta_{1}+\varepsilon
$$

but the use of Bourgain's exponent pair gives a better result.

The preceding proof is related to the method used in [14, Chapter 10] to prove that

$$
t_{n+1}-t_{n} \ll_{\varepsilon} t_{n}^{\alpha+\varepsilon}, \quad \alpha=0.15594583 \ldots,
$$

where $0<t_{1} \leqslant t_{2} \leqslant t_{3} \ldots$ are the zeros of $Z(t)$ (counted with their multiplicities), or equivalently the ordinates of zeros of $\zeta(s)$ on the "critical line" $\Re s=1 / 2$. Note that $\alpha$ in (3.10) is larger than the value $\theta=17 / 110+\varepsilon$ in 1.5 , which comes from the use of the new exponent pair 2.6 . The use of this exponent pair would yield also $\alpha=17 / 110+\varepsilon$ in 3.10 .

4. Proof of Theorem 2, The main idea of proof is the same as in the proof of Theorem 1. It suffices to consider the first bound (1.7), as the other one is proved analogously. We start from (3.1), and it will be shown that, for $k \in \mathbb{N}$ fixed

$$
\int_{T}^{T+H}\left|Z^{(k)}(t)\right| \varphi(t) \mathrm{d} t \gg H(\log T)^{k} \quad\left(T^{\varepsilon} \leqslant H \leqslant T\right)
$$


and that, with $H=T^{\theta_{k}}$ (see 1.6$)$,

$$
\int_{T}^{T+H} Z^{(k)}(t) \varphi(t) \mathrm{d} t \ll_{\varepsilon} H .
$$

From 3.3 , 4.1) and 4.2 it follows then that

$$
H \log ^{k} T \ll \int_{T}^{T+H}\left|Z^{(k)}(t)\right| \varphi(t) \mathrm{d} t \ll \int_{I_{+}^{(k)}(T, H)} Z^{(k)}(t) \varphi(t) \mathrm{d} t \leqslant H \max _{t \in I_{+}^{(k)}(T, H)} Z^{(k)}(t),
$$

which yields the first bound in (1.7). For the integral in (4.1), it may be supposed without loss of generality that $T / 2+\pi / 8=2 \pi K, K \in \mathbb{N}$, and we assume that $T^{\varepsilon} \leqslant H \leqslant T^{1 / 6}$. Setting $P=\sqrt{T /(2 \pi)}$ we have the approximate functional equation (see p. 59 of A. A. Karatsuba [21])

$$
Z^{(k)}(t)=(-1)^{k / 2} 2 \sum_{n \leqslant P}\left(\log \frac{P}{n}\right)^{k} n^{-1 / 2} \cos (t \log P-t \log n)+O\left(T^{-1 / 4} \log ^{k+1} T\right) .
$$

It can be obtained, e.g., on simplifying by Taylor's formula the expression for $Z^{(k)}(t)$ given by Theorem 5.2 of [19]. We insert 4.3) in (4.1), noting that

$$
2 \cos (t \log P-t \log n)=\mathrm{e}^{i t \log P}\left(\mathrm{e}^{-i t \log n}+\mathrm{e}^{-i t \log (T /(2 \pi n))}\right),
$$

since $P=\sqrt{T /(2 \pi)}$. This gives

$$
\begin{aligned}
\int_{T}^{T+H}\left|Z^{(k)}(t)\right| & \varphi(t) \mathrm{d} t \\
\geqslant & \int_{T}^{T+H} \varphi(t)\left|\sum_{n \leqslant P}\left(\log \frac{P}{n}\right)^{k} n^{-1 / 2}\left(\mathrm{e}^{-i t \log n}+\mathrm{e}^{-i t \log (T /(2 \pi n))}\right)\right| \mathrm{d} t \\
& +O\left(H T^{-1 / 4} \log ^{k+1} T\right) \\
\geqslant & \left|\int_{T}^{T+H} \varphi(t) \sum_{n \leqslant P}\left(\log \frac{P}{n}\right)^{k} n^{-1 / 2}\left(\mathrm{e}^{-i t \log n}+\mathrm{e}^{-i t \log (T /(2 \pi n))}\right) \mathrm{d} t\right| \\
& +O\left(H T^{-1 / 4} \log ^{k+1} T\right) .
\end{aligned}
$$

Therefore the problem reduces to the estimation of the integrals

$$
\begin{aligned}
& S_{1}:=\int_{T}^{T+H} \varphi(t) \sum_{n \leqslant P}\left(\log \frac{P}{n}\right)^{k} n^{-1 / 2} \mathrm{e}^{-i t \log n} \mathrm{~d} t \\
& S_{2}:=\int_{T}^{T+H} \varphi(t) \sum_{n \leqslant P}\left(\log \frac{P}{n}\right)^{k} n^{-1 / 2} \mathrm{e}^{-i t \log (T /(2 \pi n))} \mathrm{d} t .
\end{aligned}
$$

In $S_{1}$ the term with $n=1$ contributes

$$
\int_{T}^{T+H} \varphi(t)(\log P)^{k} \mathrm{~d} t \gg H(\log P)^{k} .
$$

Now note that, if $\alpha$ is real and $\alpha \neq 0, r$ integrations by parts give

$$
\int_{T}^{T+H} \varphi(t) \mathrm{e}^{i \alpha t} \mathrm{~d} t=\frac{(-1)^{r}}{(i \alpha)^{r}} \int_{T}^{T+H} \varphi^{(r)}(t) \mathrm{e}^{i \alpha t} \mathrm{~d} t
$$


Recall that $(3.2)$ says that

$$
\varphi^{(r)}(t) \ll_{r} H^{-r} \quad(r=0,1,2, \ldots) .
$$

In $S_{1}$ one has, for $n>1, \alpha=-\log n \gg \log 2$, and in $S_{2}$ one has

$$
\alpha=-\log (T /(2 \pi n)) \gg \log T \text {. }
$$

Thus, if $r=r(\varepsilon)$ is sufficiently large, in view of $H \geqslant T^{\varepsilon}$ and 4.4 the terms $n>1$ in $S_{1}$ and all the terms in $S_{2}$ make a contribution which is $\ll 1$, that is, negligible. This finishes the proof of 4.1 .

The formula 4.3 shows that each differentiation introduces a factor $\log P / n$ in the sum in 4.3. Thus, in view of (3.4), which is the case $k=0$ of the integral in (4.1), it seems reasonable to expect that one even has

$$
\int_{T}^{T+H}\left|Z^{(k)}(t)\right| \mathrm{d} t \gg H(\log T)^{k+1 / 4} \quad\left(T^{\varepsilon} \leqslant H \leqslant T\right) .
$$

Proving 4.5 does not seem easy.

To establish 4.2, we use 4.3. The major contribution comes from

$$
\begin{aligned}
\Re\left\{\int_{T}^{T+H} \varphi(t) \sum_{n \leqslant P}\right. & \left.\left(\log \frac{P}{n}\right)^{k} n^{-1 / 2} \exp (i t \log (P / n)) \mathrm{d} t\right\} \\
& =\Re\left\{\sum_{n \leqslant P}\left(\log \frac{P}{n}\right)^{k} n^{-1 / 2} \int_{T}^{T+H} \varphi(t) \exp (i t \log (P / n)) \mathrm{d} t\right\} .
\end{aligned}
$$

Since $P=\sqrt{T /(2 \pi)}$, on using 4.4 it is seen that the terms in 4.6 for which $|n-\sqrt{T /(2 \pi)}| \geqslant T^{1 / 2+\varepsilon} H^{-1}$ make a negligible contribution. This is similar to the discussion leading to (3.7). With $Q=[P]$ and the substitution $n=Q-m$, as in (2.1), one obtains precisely the exponential sum $(2.1)$, weighted by the monotonic factor

$$
\left(\log \frac{P}{n}\right)^{k} \ll_{k, \varepsilon} T^{\varepsilon} H^{-k},
$$

which may be conveniently removed with partial summation. What remains is an exponential sum of the type (2.1), which is estimated by 2.8 . Thus the integral in 4.2 is

$$
\ll_{\varepsilon, k} H^{1-k} T^{\varepsilon}\left(\left(T^{1 / 2} H^{-1}\right)^{2 \kappa+\lambda} T^{-\kappa / 2-1 / 4}+T^{-1 / 24}\right) \ll_{\varepsilon, k} H
$$

if $H=T^{\theta_{k}}$ with

$$
\theta_{k} \leqslant \frac{2 \kappa+2 \lambda-1}{4 k+8 \kappa+4 \lambda}+\varepsilon
$$

The value $\theta_{1}=3 / 37=0 . \overline{081}$ follows on taking

$$
(\kappa, \lambda)=(4 / 11,11 / 18)=B A(2 / 7,4 / 7) .
$$

To obtain the other value $\theta_{k} \leqslant \mu\left(\frac{1}{2}\right) / k+\varepsilon$, note that after $k$ integrations by parts the integral in 4.2 becomes

$$
(-1)^{k} \int_{T}^{T+H} \varphi^{(k)}(t) Z(t) \mathrm{d} t \ll H^{1-k} \max _{t \in[T+H]}\left|\zeta\left(\frac{1}{2}+i t\right)\right| \ll H
$$

for $H=T^{\theta_{k}}$ with $\theta_{k} \leqslant \mu\left(\frac{1}{2}\right) / k+\varepsilon$, as asserted. This completes the proof of Theorem 2 
5. Proof of Theorem 3. First note that, under the hypotheses of Theorem 3

$$
\left|\zeta\left(\frac{1}{2}+i t\right)\right|=\left|\int_{T}^{t} Z^{\prime}(t) \mathrm{d} t\right| \leqslant\left|\int_{T}^{T+H} \psi(t) Z^{\prime}(t) \mathrm{d} t\right| .
$$

Here $\psi(t)(\geqslant 0)$ is a smooth function constructed in the following way. If $t \in[T, T+3 H / 4]$, then $\psi(t)=1$, and $\psi(t)=0$ for $t \geqslant T+H$. Moreover, $\psi^{\prime}(t) \ll H^{-1}$. The second integral in $(5.1)$ is

$$
-\int_{T}^{T+H} \psi^{\prime}(t) Z(t) \mathrm{d} t
$$

and this leads again to the exponential sum in 2.1), weighted by the factor $\psi^{\prime}(t) \ll 1 / H$. Using again 2.8 we have

$$
\begin{aligned}
\zeta\left(\frac{1}{2}+i t\right) & \ll_{\varepsilon} T^{\varepsilon}\left(\left(T^{1 / 2} H^{-1}\right)^{2 \kappa+\lambda} T^{-\kappa / 2-1 / 4}+T^{-1 / 24}\right) \\
& \ll_{\varepsilon} T^{(\kappa+\lambda) / 2-1 / 4+\varepsilon} H^{-2 \kappa-\lambda}+T^{\varepsilon-1 / 24} .
\end{aligned}
$$

Therefore we obtain $(1.8)$ and $(1.9)$ with the exponent pair $(2.6)$. This completes the proof of Theorem 3 It is similar to the second part of Theorem 2 in [15]. The condition $\zeta\left(\frac{1}{2}+i T\right)=0$ is not so restrictive, as if this happens, there is a $H_{0}(>0)$ such that $Z(t)$ is monotonic in $\left[T, T+H_{0}\right]$. In fact, it is well known that under the Riemann Hypothesis $(\mathrm{RH})$ the zeros of $Z(t)$ and $Z^{\prime}(t)$ are interlacing, namely that $Z^{\prime}(t)$ has exactly one zero between two consecutive zeros of $Z(t)$ (see e.g., H. M. Edwards [8]). R. J. Anderson [1] showed that the zeros of $Z^{\prime}(t)$ and $Z^{\prime \prime}(t)$ are also interlacing $(\mathrm{RH})$. More recently K. Matsuoka 23. showed that the zeros of $Z^{(n)}(t)$ and $Z^{(n+1)}(t)$ are also interlacing for $n=2,3, \ldots(\mathrm{RH})$. But unconditionally, what can one say about the number of zeros of $Z^{\prime}(t)$ between two consecutive zeros $t_{n}, t_{n+1}$ of $Z(t)$ ? If the number of such zeros is denoted by $M(n)$, is it true that $M(n)=O(\log n)$ ? The analogous question can be asked about the zeros of $Z^{(k)}(t)$ in general. R. J. Anderson [1] proved that

$$
N^{(1)}(T) \leqslant \frac{T}{2 \pi} \log \frac{T}{2 \pi}-\frac{T}{2 \pi}+O(\log T),
$$

where $N^{(1)}(T)$ denotes the number of zeros of $Z^{\prime}(t)$ in $[0, T]$. Moreover, if the RH holds, then he showed that equality holds in 5.3 . If equality holds unconditionally, this would imply that

$$
\limsup _{n \rightarrow \infty} \frac{M(n)}{\log n}=+\infty
$$

cannot hold.

6. Some remarks. In this section some remarks pertaining to Theorem 1 and related topics are presented.

REMARK 1. One can prove without difficulty that

$$
\max _{T \leqslant t \leqslant T+H, Z(t)>0} Z(t) \gg(\log T)^{1 / 4} \quad\left(T^{1 / 4} \leqslant H \leqslant T\right),
$$

and similarly

$$
\max _{T \leqslant t \leqslant T+H, Z(t)<0}-Z(t) \gg(\log T)^{1 / 4} \quad\left(T^{1 / 4} \leqslant H \leqslant T\right) .
$$


The bounds are the same as in (1.3) and $(1.4)$, but the range for $H$ is poorer. To see this, note that we have (this corresponds to $\varphi(t) \equiv 1, t \in[T, T+H]$ in 3.1 for $k=0$ )

$$
\int_{T, Z(t)>0}^{T+H} Z(t) \mathrm{d} t=\frac{1}{2}\left(\int_{T}^{T+H} Z(t) \mathrm{d} t+\int_{T}^{T+H}|Z(t)| \mathrm{d} t\right),
$$

and similarly

$$
\int_{T, Z(t)<0}^{T+H} Z(t) \mathrm{d} t=\frac{1}{2}\left(\int_{T}^{T+H} Z(t) \mathrm{d} t-\int_{T}^{T+H}|Z(t)| \mathrm{d} t\right) .
$$

Let now $T^{1 / 4} \leqslant H \leqslant T$. Then from

$$
\int_{0}^{T} Z(t) \mathrm{d} t=O\left(T^{1 / 4}\right)
$$

we get

$$
\int_{T}^{T+H} Z(t) \mathrm{d} t=O\left(T^{1 / 4}\right) .
$$

The bound in (6.3) was proved independently by M. Jutila [20] and M. Korolev [22], who used different methods in their proofs. It follows that

$$
\int_{T, Z(t)>0}^{T+H} Z(t) \mathrm{d} t \gg H(\log T)^{1 / 4} \quad\left(T^{1 / 4} \leqslant H \leqslant T\right),
$$

and one obtains 6.1. The proof of 6.2 is analogous.

Remark 2. The bounds in (1.4) are much weaker than the ones in (1.1) and (1.2). However, Theorem 1 provides good localization of large positive and small negative values of $Z(t)$. An improvement of the power of the logarithm in Theorem 1 would follow e.g., if one had good upper bounds for higher odd moments of $Z(t)$. At present, nothing but trivial bounds is known (see the monograph [19]). For example, the best one can currently get for the cubic moment of $Z(t)$ is

$$
\left|\int_{0}^{T} Z^{3}(t) \mathrm{d} t\right| \leqslant \int_{0}^{T}\left|Z^{3}(t)\right| \mathrm{d} t=\int_{0}^{T}\left|\zeta\left(\frac{1}{2}+i t\right)\right|^{3} \mathrm{~d} t \ll T(\log T)^{9 / 4} .
$$

The last bound is a recent result of S. Bettin, V. Chandee and M. Radziwiłł [3].

It was stated by the author in [19] that it is plausible that one has

$$
\int_{0}^{T} Z^{3}(t) \mathrm{d} t=O_{\varepsilon}\left(T^{3 / 4+\varepsilon}\right) .
$$

In fact, it is known (see equation (11.9) of [19]) that

$$
\int_{T}^{2 T} Z^{3}(t) \mathrm{d} t=2 \pi \sqrt{\frac{2}{3}} \sum_{(T / 2 \pi)^{3 / 2} \leqslant n \leqslant(T / \pi)^{3 / 2}} d_{3}(n) n^{-1 / 6} \cos \left(3 \pi n^{2 / 3}+\frac{1}{8} \pi\right)+O_{\varepsilon}\left(T^{3 / 4+\varepsilon}\right),
$$

where $d_{3}(n)$ (generated by $\zeta^{3}(s)$ ) is the number of ways $n$ can be represented as a product of three natural numbers. Thus the problem of evaluating the cubic moment of $Z(t)$ reduces to the estimation of the exponential sum on the right-hand side of the above equation. 
Unconditionally it is known (see K. Ramachandra [25]) that

$\int_{T}^{T+H}|Z(t)|^{3} \mathrm{~d} t=\int_{T}^{T+H}\left|\zeta\left(\frac{1}{2}+i t\right)\right|^{3} \mathrm{~d} t \gg H(\log H)^{9 / 4} \quad(\log \log T \ll H \leqslant T)$.

Using (6.4), 6.5 and an argument similar to the one above it would follow that

$$
\begin{aligned}
\int_{T, Z(t)>0}^{T+H} Z^{3}(t) \mathrm{d} t & =\frac{1}{2}\left(\int_{T}^{T+H}|Z(t)|^{3} \mathrm{~d} t+\int_{T}^{T+H} Z^{3}(t) \mathrm{d} t\right) \\
& \gg H(\log T)^{9 / 4} \quad\left(T^{3 / 4+\varepsilon} \leqslant H \leqslant T\right) .
\end{aligned}
$$

This yields

$$
\max _{T \leqslant t \leqslant T+H, Z(t)>0} Z(t) \gg(\log T)^{3 / 4} \quad\left(T^{3 / 4+\varepsilon} \ll H \leqslant T\right),
$$

and the analogous lower bound

$$
\max _{T \leqslant t \leqslant T+H, Z(t)<0}-Z(t) \gg(\log T)^{3 / 4} \quad\left(T^{3 / 4+\varepsilon} \ll H \leqslant T\right) .
$$

REMARK 3. Another possibility, similar to the one above, is to try proving the bound

$$
\int_{T}^{2 T} Z(t)|Z(t)| \mathrm{d} t=O(T)
$$

which seems to be of independent interest. There is massive cancellation in the primitive of $Z(t)$, as witnessed by (6.3). Thus it seems plausible that (6.6) should hold (or even a stronger bound). We have

$$
\begin{aligned}
\int_{T}^{2 T} Z(t)|Z(t)| \mathrm{d} t & =\int_{T, Z(t)>0}^{2 T} Z(t)|Z(t)| \mathrm{d} t+\int_{T, Z(t)<0}^{2 T} Z(t)|Z(t)| \mathrm{d} t \\
& =\int_{T, Z(t)>0}^{2 T}\left|\zeta\left(\frac{1}{2}+i t\right)\right|^{2} \mathrm{~d} t-\int_{T, Z(t)<0}^{2 T}\left|\zeta\left(\frac{1}{2}+i t\right)\right|^{2} \mathrm{~d} t .
\end{aligned}
$$

Similarly we obtain

$$
\int_{T}^{2 T}\left|\zeta\left(\frac{1}{2}+i t\right)\right|^{2} \mathrm{~d} t=\int_{T, Z(t)>0}^{2 T}\left|\zeta\left(\frac{1}{2}+i t\right)\right|^{2} \mathrm{~d} t+\int_{T, Z(t)<0}^{2 T}\left|\zeta\left(\frac{1}{2}+i t\right)\right|^{2} \mathrm{~d} t .
$$

Adding these relations we infer that

$$
\begin{aligned}
\int_{T, Z(t)>0}^{2 T}\left|\zeta\left(\frac{1}{2}+i t\right)\right|^{2} \mathrm{~d} t & =\frac{1}{2}\left(\int_{T}^{2 T}\left|\zeta\left(\frac{1}{2}+i t\right)\right|^{2} \mathrm{~d} t+\int_{T}^{2 T} Z(t)|Z(t)| \mathrm{d} t\right) \\
& =\frac{1}{2} T \log T+O(T),
\end{aligned}
$$

where we used 6.6 and the elementary formula

$$
\int_{0}^{T}\left|\zeta\left(\frac{1}{2}+i t\right)\right|^{2} \mathrm{~d} t=T \log T+O(T)
$$

We obtain

$$
\frac{1}{2} T \log T+O(T) \leqslant T \max _{T \leqslant t \leqslant 2 T, Z(t)>0} Z^{2}(t) .
$$

This implies

$$
\max _{T \leqslant t \leqslant 2 T, Z(t)>0} Z(t) \geqslant \sqrt{\frac{1}{2} \log T+O(1)},
$$


and a similar bound for $-Z(t)$ when $Z(t)<0$. Of course, the problem of proving 6.6 remains. We note that 6.6 is equivalent to

$$
\int_{T, Z(t)>0}^{2 T}\left|\zeta\left(\frac{1}{2}+i t\right)\right|^{2} \mathrm{~d} t=\frac{1}{2} T \log T+O(T) .
$$

REMARK 4. Let $0<\gamma_{1} \leqslant \gamma_{2} \leqslant \gamma_{3} \leqslant \ldots$ denote ordinates of complex zeros of the Riemann zeta-function, with multiplicities counted. If the Riemann Hypothesis holds, then

$$
\left\{\gamma_{n}\right\}_{n=1}^{\infty} \equiv\left\{t_{n}\right\}_{n=1}^{\infty} .
$$

However, for the difference $t_{n+1}-t_{n}$ only the (unconditional) bound 3.10 is known. For $\gamma_{n+1}-\gamma_{n}$ a much better bound holds, which is to be expected, since there are at least as many $\gamma_{n}$ 's as there are $t_{n}$ 's in any finite interval. Namely from Theorem 9.12 of E. C. Titchmarsh [27] it follows that unconditionally, for some $A>0, n \geqslant n_{0}$,

$$
\gamma_{n+1}-\gamma_{n} \leqslant \frac{A}{\log \log \log \gamma_{n}} .
$$

R. R. Hall and W. K. Hayman [8] showed that any constant $A>\pi / 2$ is permissible in 6.7 .

REMARK 5. The result of Theorem 1 is unconditional. However, it seems difficult to obtain sharper lower bounds even if one assumes the Riemann Hypothesis.

REMARK 6. In the author's papers [17], [18] small values of the zeta-function on the critical line and gaps between consecutive zeros are investigated. A conditional result, on the RH and the so called Gaussian unitary ensemble hypothesis (GUE), states that for almost all $\gamma_{n}\left(\equiv t_{n}\right)$

$$
\max _{\gamma_{n} \leqslant t \leqslant \gamma_{n+1}}|Z(t)| \equiv \max _{\gamma_{n} \leqslant t \leqslant \gamma_{n+1}}\left|\zeta\left(\frac{1}{2}+i t\right)\right| \geqslant \gamma_{n+1}-\gamma_{n} .
$$

In other words, most of the time the maximum of $|Z(t)|$ between its two consecutive zeros is larger than the gap between these zeros.

The GUE hypothesis states that, on the RH and with

$$
0 \leqslant \alpha<\beta<\infty, \quad \delta_{n}=\frac{1}{2 \pi}\left(\gamma_{n+1}-\gamma_{n}\right) \log \left(\frac{\gamma_{n}}{2 \pi}\right),
$$

we have

$$
\sum_{\gamma_{n} \leqslant T, \delta_{n} \in[\alpha, \beta]} 1=\left(\int_{\alpha}^{\beta} p(0, u) \mathrm{d} u+o(1)\right) \frac{T}{2 \pi} \log \left(\frac{T}{2 \pi}\right) \quad(T \rightarrow \infty) .
$$

The function $p(0, u)$ (and the related function $p(k, u)$ ) is a certain probabilistic density, given by complicated functions defined in terms of linear prolate spheroidal wave functions. We have

$$
\begin{gathered}
1-\left(\frac{\sin \pi u}{\pi u}\right)^{2}=\sum_{k=0}^{\infty} p(k, u), \\
p(0, u)=\frac{1}{3} \pi^{3} u^{2}-\frac{2}{15} \pi^{4} u^{4}+\frac{1}{315} \pi^{6} u^{6}+\ldots \quad(u \rightarrow 0+),
\end{gathered}
$$


and

$$
\log p(0, u)=-\frac{\pi^{2}}{8}+o(1) \quad(u \rightarrow \infty) .
$$

The asymptotic formula 6.8 is to be compared with the classical Riemann-von Mangoldt formula (see e.g., Chapter 1 of [14])

$$
\sum_{\gamma_{n} \leqslant T} 1=\frac{T}{2 \pi} \log \left(\frac{T}{2 \pi}\right)-\frac{T}{2 \pi}+O(\log T) .
$$

REMARK 7. If one considers large values not of $Z(t)$, but of its primitive, namely

$$
F(t):=\int_{0}^{t} Z(u) \mathrm{d} u
$$

then the following result holds. There exist positive constants $A, B$ such that every interval $[x, x+A \sqrt{x}]$, for $x \geqslant x_{0}>0$, contains two points $x_{1}, x_{2}$ for which

$$
F\left(x_{1}\right)>B x_{1}^{1 / 4}, \quad F\left(x_{2}\right)<-B x_{2}^{1 / 4} .
$$

The omega-results in 6.9 follow from Theorem 1 of M. A. Korolev [22] with

$$
x=2 \pi N^{2}, \quad A>2 \pi \sqrt{2 \pi}, \quad \vartheta=\frac{1}{2},
$$

and $N$ even or odd, respectively.

\section{References}

[1] R. J. Anderson, On the function $Z(t)$ associated with the Riemann zeta-function, J. Math. Anal. Appl. 118 (1986), 323-340.

[2] R. Balasubramanian, On the frequency of Titchmarsh's phenomenon for $\zeta(s)$ IV, HardyRamanujan J. 9 (1986), 1-10.

[3] S. Bettin, V. Chandee, M. Radziwiłł, The mean square of the product of the Riemann zeta function with Dirichlet polynomials, J. Reine Angew. Math. 729 (2017), 51-79.

[4] E. Bombieri, H. Iwaniec, On the order of $\zeta\left(\frac{1}{2}+i t\right)$, Ann. Scuola Norm. Sup. Pisa Cl. Sci. (4) 13 (1986), 449-472.

[5] E. Bombieri, H. Iwaniec, Some mean value theorems for exponential sums, Ann. Scuola Norm. Sup. Pisa Cl. Sci. (4) 13 (1986), 473-486.

[6] A. Bondarenko, K. Seip, Large greatest common divisor sums and extreme values of the Riemann zeta function, Duke Math. J. 166 (2017), 1685-1701.

[7] J. Bourgain, Decoupling, exponential sums and the Riemann zeta-function, J. Amer. Math. Soc. 30 (2017), 205-224.

[8] H. Edwards, Riemann's Zeta Function, Pure and Appl. Math. 58, Academic Press, New York, 1974.

[9] S. M. Gonek, A. Ivić, On the distribution of positive and negative values of Hardy's Z-function, J. Number Theory 174 (2017), 189-201.

[10] R. R. Hall, W. K. Hayman, Hyperbolic distance and distinct zeros of the Riemann zetafunction in small regions, J. Reine Angew. Math. 526 (2000), 35-59.

[11] M. N. Huxley, Area, Lattice Points and Exponential Sums, London Math. Soc. Monogr. (N.S.) 13, Oxford Science Publ., Clarendon Press, New York, 1996.

[12] M. N. Huxley, Exponential sums and lattice points III, Proc. London Math. Soc. (3) 87 (2003), 591-609. 
[13] M. N. Huxley, Exponential sums and the Riemann zeta function V, Proc. London Math. Soc. (3) 90 (2005), 1-41.

[14] A. Ivić, The Riemann Zeta-Function, Wiley, New York 1985 (reissue, Dover, Mineola, New York, 2003).

[15] A. Ivić, On a problem connected with zeros of $\zeta(s)$ on the critical line, Monatsh. Math. 104 (1987), 17-27.

[16] A. Ivić, Mean Values of the Riemann Zeta Function, Tata Inst. Fund. Res. Lectures on Math. and Phys. 82, Springer, Berlin, 1991; www.math.tifr.res.in/ publ/ln/tifr82.pdf

[17] A. Ivić, On sums of gaps between the zeros of $\zeta(s)$ on the critical line, Univ. Beograd. Publ. Elektrotehn. Fak. Ser. Mat. 6 (1995), 55-62.

[18] A. Ivić, On small values of the Riemann zeta-function on the critical line and gaps between zeros, Liet. Mat. Rink. 42 (2002), 31-45; transl.: Lithuanian Math. J. 42 (2002), 25-36.

[19] A. Ivić, The Theory of Hardy's Z-function, Cambridge Tracts in Math. 196, Cambridge Univ. Press, Cambridge, 2013.

[20] M. Jutila, An asymptotic formula for the primitive of Hardy's function, Ark. Mat. 49 (2011), 97-107.

[21] A. A. Karatsuba, On the distance between consecutive zeros of the Riemann zeta-function on the critical line (in Russian), Trudy Mat. Inst. Steklov. 157 (1981), 49-63.

[22] M. A. Korolev, On the integral of Hardy's function $Z(t)$ (in Russian), Izv. Ross. Akad. Nauk Ser. Mat. 72, no. 3, 19-68; English transl.: Izv. Math. 72 (2008), 429-478.

[23] K. Matsuoka, On the higher derivatives of $Z(t)$ associated with the Riemann zeta-function, arXiv: 1205.2161

[24] H. L. Montgomery, Extreme values of the Riemann zeta function, Comment. Math. Helv. 52 (1977), 511-518.

[25] K. Ramachandra, On the Mean-Value and Omega-Theorems for the Riemann ZetaFunction, Tata Inst. Fund. Res. Lectures on Math. and Phys. 85, Springer, Berlin, 1995; to be found online at www.math.tifr.res.in/ $\mathrm{publ} / \mathrm{ln} / \mathrm{tifr} 85 . \mathrm{pdf}$

[26] K. Soundararajan, Extreme values of zeta and L-functions, Math. Ann. 342 (2008), 467-486.

[27] E. C. Titchmarsh, The Theory of the Riemann Zeta-Function, 2nd ed. edited by D. R. Heath-Brown, Clarendon Press, New York, 1986. 
